\title{
Mosquito Host-Seeking Diel Rhythm and Chemosensory Gene Expression is Affected by Age and Plasmodium Stages
}

Melika Hajkazemian

Stockholm University

Sharon R. Hill

Swedish University of Agricultural Sciences

Raimondas Mozūraitis

Stockholm University

Lisa Ranford-Cartwright

University of Glasgow

S. Noushin Emami ( $\nabla$ noushin.emami@su.se )

Stockholm University

Rickard Ignell

Swedish University of Agricultural Sciences

\section{Research Article}

Keywords: Plasmodium falciparum, parasite stages, Anopheles gambiae, vector, locomotion, olfaction, antennae, transcriptomics

Posted Date: December 9th, 2021

DOI: https://doi.org/10.21203/rs.3.rs-1114857/v1

License: (c) (1) This work is licensed under a Creative Commons Attribution 4.0 International License. Read Full License 


\section{Abstract}

Malaria parasites can affect vector-related behaviours, increasing transmission success. Using Anopheles gambiae and Plasmodium falciparum, we consider the effect of interaction between infection stage and vector age on diel locomotion in response to human odour and the expression of antennal chemosensory genes. We demonstrate age-dependent behavioural diel compartmentalisation by uninfected females. Infection disrupts overall and diel activity patterns compared with age-matched controls. Mosquitoes carrying transmissible sporozoites are more active, shifting activity periods to coincide with human host availability, in response to human odour. Older, uninfected females reduce activity during their peak host-seeking period in response to human odour. Age- and infection stagespecific changes in odour-mediated locomotion coincide with altered transcript abundance of select chemosensory genes providing a possible molecular mechanism regulating the behaviour. Our results support the hypothesis that vector-related behaviours of female mosquitoes are altered by infection stage and further modulated by the age of the vector, and have important implications for malaria transmission and disease dynamics.

\section{Summary}

The presence of different life-cycle stages of the malaria parasite, Plasmodium falciparum, together with the age of the vector, affect the host-seeking daily rhythm and antennal chemosensory gene expression of Anopheles gambiae to indirectly increase the odds of successful disease transmission.

\section{Introduction}

Malaria parasites depend on mosquito vectors for transmission from one host to another. Increasing empirical evidence demonstrates that insect-borne pathogens may manipulate both their human host and their insect vector to enhance transmission success ${ }^{1-4}$. This manipulation is not static, as vectorrelated behaviours can vary significantly between uninfected and infected vectors over different time scales relevant to the disease transmission dynamics ${ }^{3-5}$. Pathogens are known to affect vector behaviours, including locomotion, flight, host seeking, probing and feeding, which can manifest in increased avidity to locate and obtain a blood meal or in alterations in host preference, resulting in an enhanced transmission through the increased contact between vector and host ${ }^{3,5,6}$. The molecular mechanisms by which these behavioural traits may be modulated have only recently received attention, identifying pathways in the neuroendocrine circuitry as key targets ${ }^{2,5,7,8}$.

Mosquitoes acquire Plasmodium parasites, the causative agent of malaria, through a blood meal from an infected vertebrate host. The pathogen undergoes several developmental stages in the mosquito, from midgut invasion and oocyst formation (infected mosquitoes, 7 days post-infection; dpi) through to the invasion of the salivary glands, when a mosquito can transmit the malaria sporozoites (infectious mosquitoes, $14 \mathrm{dpi})^{9}$. The avidity of mosquitoes to engage in vector-related behaviours, including host seeking and blood feeding, depends on the developmental stage of the pathogen in the mosquito, rather 
than the infection status of the mosquito alone $e^{3}$. Available data for Plasmodium parasites that cause avian, rodent and human malaria emphasise that vector-related behaviours are suppressed when mosquitoes are infected, but not able to transmit the pathogen, whereas infectious mosquitoes are more active $\mathrm{e}^{10-12}$. This modulation appears to be conserved across vectors and other pathogens, e.g., Aedes aegypti that transmit dengue and Zika virus ${ }^{5,13}$. The observed changes in the odour-mediated hostseeking behaviour, demonstrated for both infected and infectious mosquitoes, are reflected in the selective sensitivity of the peripheral olfactory system to key host-related volatile organic compounds $5,10,14$. Such modulation may be regulated through alterations in neural signalling and chemosensory gene expression, as demonstrated in malaria- and dengue-infected mosquitoes ${ }^{2,5}$.

In depth analysis of the molecular basis of olfaction in mosquitoes, has allowed for the identification and functional characterisation of chemosensory gene families, including odorant receptors (Ors), gustatory receptors (Grs), ionotropic receptors (Irs), odorant binding proteins (Obps) and chemosensory proteins $(C s p s)^{15-19}$. The expression of select members of these gene families in olfactory-related tissues change in response to adult maturation, age and physiological state ${ }^{20-25}$, including infection status ${ }^{5}$. These state-dependent changes in gene abundance coincide with concerted alterations in the sensitivity of olfactory sensory neurones and the behaviour of the mosquitoes $20,21,25$.

This study analysed the stage-dependent effect of $P$. falciparum infection on the locomotion activity of female Anopheles gambiae in the presence and absence of human odour, demonstrating that infected and infectious mosquitoes display differential locomotor activity correlating with age, infection status and time of day. The potential molecular mechanisms underlying the age- and infection-dependent odourmediated locomotor activity were assessed by transcriptome analysis, identifying differentially abundant chemosensory-related genes in the antennae coinciding with the demonstrated differences in mosquito behaviour. A better understanding of the effect of infection on locomotor activity and odour-mediated host seeking throughout the day has the potential to more accurately direct vector measures. Identifying the molecular mechanisms by which these behavioural traits are modulated by $P$. falciparum may lead to a better understanding of vectorial capacity and transmission dynamics potentially inspiring the development of innovative control strategies.

\section{Results}

\section{Mosquito age and the stage of Plasmodium falciparum infection affect locomotor activity}

Locomotor activity of uninfected female An. gambiae in the absence of human odour significantly reduced with age when assessed in Drosophila activity monitors $\left(\chi^{2}{ }_{1}=5.86, p=0.02\right.$; Fig. $\left.1 \mathrm{a}-\mathrm{b}\right)$. This age effect was not observed following infection with $P$. falciparum $\left(\chi^{2}{ }_{1}=0.83 p=0.35\right.$; Fig. $\left.1 \mathrm{~b}\right)$. In the presence of human odour, the age-dependent reduction in locomotor activity was observed in both uninfected $\left(\chi^{2}{ }_{1}=21.12, p<0.001\right)$ and $P$. falciparum carrying mosquitoes $\left(\chi^{2}{ }_{1}=5.73, p=0.02\right.$; the GLMM:Imer model was constrained by age and the random effect of experimental replication; Fig. 1b). 
Infection status significantly affected the activity of mosquitoes when comparing the overall locomotion profiles of $7 \mathrm{dpi}$ and $14 \mathrm{dpi}$ individuals with age-matched uninfected females in the presence and absence of human odour ( $\beta$-estimated $=$ weighted mean value based on all variables plus the random effect of the replication \pm SE; presence of human odour: $\chi^{2}{ }_{1}=18.06, p<0.001$; absence of human odour: $\left.\chi^{2}{ }_{1}=5.73, p<0.01\right)$. In the absence of human odour, $7 \mathrm{dpi}$ females were less active compared to agematched controls $\left(\chi^{2}{ }_{1}=8.21, p=0.004\right)$, whereas 14 dpi females were as active as their age-matched controls $\left(\chi^{2}{ }_{1}=2.27, p=0.13\right.$; Fig. $\left.1 \mathrm{c}\right)$. The presence of human odour differentially altered the locomotor activity. While there were no significant differences between $7 \mathrm{dpi}$ and age-matched females in the presence of human odour $\left(\chi^{2}{ }_{1}=0.12, p=0.73\right)$, the infectious $14 \mathrm{dpi}$ mosquitoes were more active than age-matched uninfected females (14 days control; $14 \mathrm{dc} \chi^{2}{ }_{1}=8.80, p=0.002$; Fig. $1 \mathrm{c}$ ).

\section{Mosquito locomotor activity varies temporally in response to host odour and P. falciparum infection}

Diurnal cycles in An. gambiae locomotor and host-seeking activities were evident, with distinct crepuscular activity peaks culminating in the highest activity at the onset of photophase (ZT 23-24), and low to moderate activity during scotophase (ZT 12-23) and low activity during photophase (ZT 0-11) ${ }^{26}$. To assess the diurnal effect on the locomotor activity of mosquitoes with $P$. falciparum infection and their respective controls, a mixed model analysis was used (Imer model: constrained by two main explanatory variables, infection status and period of time, as well as the random effect of replication, Supplementary Table 1-2). In the absence of human odour, the diurnal locomotor activity profile of uninfected mosquitoes confirmed the high activity at dawn (ZT 23-24), with the younger cohort being more active during scotophase with a peak at dawn, whereas the older control females displayed an increased activity at dusk that was maintained until dawn (ZT 0-11: $\chi^{2}{ }_{1}=27.43, p<0.001 ; Z T$ 11-12: $\chi^{2}{ }_{1}=4.58, p=$ 0.049; ZT 12-23: $\chi^{2}{ }_{1}=4.33, p=0.03 ;$ ZT 23-24: $\chi^{2}{ }_{1}=32.32, p<0.001$, Fig. 1 d, Supplementary Table 2.). In the presence of human odour, the locomotor activity of the younger uninfected cohort increased at dawn, and remained at this activity level until dusk, while the older females only displayed an increase in activity at dusk (ZT 0-11: $\chi^{2}{ }_{1}=17.54, p<0.001 ; Z T$ 11-12: $\chi^{2}{ }_{1}=7.67, p=0.005 ; Z T$ 12-23: $\chi^{2}{ }_{1}=15.21, p<0.001$; ZT 23-24: $\chi^{2}{ }_{1}=18.25, p<0.001$, Fig. $1 \mathrm{~d}$, Supplementary Table 2.).

The time-of-day, and the stage of $P$. falciparum sporogony, differentially affected the locomotor activity of the vector (Fig. 1d). In mosquitoes with parasites undergoing sporogony, there was no effect of age on locomotion throughout the $24 \mathrm{~h}$ period in the absence of human odour (ZT 11-12: $\chi^{2}{ }_{1}=1.71, p=0.18 ; \mathrm{ZT}$ 12-23: $\chi^{2}{ }_{1}=0.31, p=0.57 ; \mathrm{ZT} 23-24: \chi^{2}{ }_{1}=3.49, p=0.06$, Supplementary Table 2.), except during photophase, in which infectious (14 dpi) mosquitoes demonstrated lower activity than infected ( $7 \mathrm{dpi})$ individuals (ZT 0-11: $\left.\chi^{2}{ }_{1}=21.97, p<0.001\right)$. However, in the presence of human odour, the infectious cohort (14 dpi) was more active at dawn (ZT 23-24: $\left.\chi^{2}{ }_{1}=15.40, p<0.001\right)$, while being less active than the younger mosquitoes throughout the rest of the diurnal period (ZT $0-11: \chi^{2}{ }_{1}=9.35, p=0.002 ; Z T 11-12$ : $\chi^{2}{ }_{1}=20.02, p<0.001 ;$ ZT 12-23: $\chi^{2}{ }_{1}=9.73, p=0.001$, Supplementary Table 2.). 
Individual locomotor profiles of mosquitoes at $7 \mathrm{dpi}$ and $14 \mathrm{dpi}$ differed significantly from that of agematched uninfected controls (Fig. 1d). Infection significantly reduced the locomotor activity in $7 \mathrm{dpi}$ females in the absence of human odour when compared with their uninfected counterparts during most time periods (ZT 0-11: $\chi^{2}{ }_{1}=8.49, p=0.03 ; \mathrm{ZT} \mathrm{11-12:} \chi^{2}{ }_{1}=1.008, p=0.31 ; \mathrm{ZT} 12-23: \chi^{2}{ }_{1}=7.84, p=0.005$; ZT 23-24: $\chi^{2}{ }_{1}=13.78, p<0.001$, Supplementary Table 1-2). Mosquitoes carrying the transmissible stage of the parasite ( $14 \mathrm{dpi}$ ) displayed a lower locomotor activity than the uninfected counterparts throughout photophase and scotophase (ZT 0-11: $\chi^{2}{ }_{1}=5.92, p=0.01 ;$ ZT 12-23: $\chi^{2}{ }_{1}=11.87, p<0.001$,

Supplementary Table 1-2), but not at dusk and dawn (ZT 11-12: $\chi^{2}{ }_{1}=2.68, p=0.10 ; ; Z \mathrm{ZT} 23-24: \chi^{2}{ }_{1}=2.20$, $p=0.10)$. In the presence of human odour, the infected (7 dpi) females showed lower activity than agematched controls at dawn (ZT 23-24: $\left.\chi^{2}{ }_{1}=7.29, p=0.006\right)$, while the inverse activity pattern was observed during photophase (ZT 0-11: $\left.\chi^{2}{ }_{1}=12.28, p=0.001\right)$. The infectious mosquitoes (14 dpi) demonstrated a significantly increased locomotor activity at dawn (ZT 23-24: $\left.\chi^{2}{ }_{1}=23.78, p<0.001\right)$ and during scotophase (ZT 12-23: $\chi^{2}{ }_{1}=25.42, p=0.001$ ), while there was no difference in activity of the infectious and uninfected females during photophase (ZT 0-11: $\chi^{2}{ }_{1}=2.26, p=0.13$ ), with a significant reduction in dusk (ZT 11-12: $\chi^{2}{ }_{1}=16.06, p<0.001$, Supplementary Table $\left.1-2\right)$.

\section{Plasmodium falciparum modulates antennal transcript abundance}

Paired-end sequencing of each of the libraries constructed from antennal RNA, with a total of 2400 antennae, generated an average mapping of 26399740 million cleaned reads per library. Out of the 13 832 coding genes annotated in the genome of An. gambiae (Agam4.10), a total of 10115 transcripts were reliably detected above 1 transcript per million (TPM) mapped reads in the antennae, among all experimental and control groups, demonstrating an adequate level of coverage.

A principal component analysis (PCA) of the antennal transcripts was conducted to demonstrate the overall variation among the antennal transcriptomes (infected, infectious, and age-matched uninfected conditions; 4 replicates each; Fig. 2a). The PCA identified that $49.8 \%$ of the variation among the libraries was based on the relative infection status in each age group (PC1), while $15.3 \%$ of the variance was dependent on age and infection status relative to the controls (PC2; Fig. 2a). All of the biological replicates of the same age and infection status clustered tightly together in the principal component space, except for the infectious samples (14 dpi), demonstrating that variation in the libraries due to handling and processing was successfully minimised. The separation of the four libraries of the $14 \mathrm{dpi}$ samples into two clusters correlates with demonstrated differences in parasite load of the mosquitoes (Fig. 2a; Supplementary Fig. 1).

Antennal transcripts were significantly differentially regulated between the two age-matched cohorts (6 187), of which 3465 were differentially abundant only between $14 \mathrm{dpi}$ and $14 \mathrm{dc}$, whereas in $7 \mathrm{dpi}$ and 7 dc, 850 were differentially abundant (Fig. 2b). In total, 4807 transcripts were significantly differentially regulated between the two age-matched control groups and the two groups carrying $P$. falciparum parasites, of which 1071 transcripts were shared between them (Fig. 2c). Within the age-matched control 
groups, 2614 transcripts were uniquely regulated, whereas 1122 were uniquely regulated within the two groups carrying $P$. falciparum parasites (Fig. 2c; Supplementary Fig. 2). Overall, differentially abundant transcripts were not condition-dependently regulated, except those regulated post-infection during both the infected and infectious stages (1 872), of which more than $80 \%$ of the transcripts were downregulated post-infection (Fig. 2b).

To characterise the functional ontology of the differentially abundant genes in the antennae of infected and infectious mosquitoes with their age-matched controls, a gene ontology (GO) analysis of molecular function (level three) was conducted (Fig. 3). Of the 3685 differentially abundant transcripts between the two uninfected control groups, the majority ( $>75 \%)$ were functionally classified as structural constituent of cuticle (GO: 0042302) and enzyme inhibitor activity (GO: 0004857; Fig. 3a). Both of these classes were more abundant in older compared to the younger individuals. None of the age-dependent $\mathrm{GO}$ terms identified in the uninfected cohort comparison (Fig. 3a) were detected in the pairwise comparisons of the antennal transcriptomes among infected, infectious and their age-matched controls. The three most represented functional classes in the pairwise comparisons between the infected and infectious groups with their age-matched controls, as well as between the infected and infectious antennal transcriptomes, were heterocyclic compound binding (GO: 1901363), organic cyclic compound binding (GO: 0097159) and ion binding (GO: 0043167; Fig. 3b). Two functional classes were regulated differently in the antenna of mosquitoes with a $P$. falciparum infection, odorant binding (GO: 0005549) and carbohydrate derivative binding (G0:0097367). The number of genes in the functional class odorant binding were regulated in both infected and infectious samples, while those in the carbohydrate derivative binding class were differentially regulated only in infectious samples (Fig. 3b).

A detailed analysis of the major chemosensory gene families associated with the odorant binding functional class, Ors, Irs, Grs, Csps and Obps, was conducted. As the mosquitoes aged, $34 \%$ of the chemosensory genes were significantly regulated in the antennae of uninfected females, with chemosensory receptors demonstrating higher abundance in the older females, while the binding proteins were both up- and down-regulated (Fig. 4). Following P. falciparum infection, the abundance of 18 Obps reduced with age, while only one chemosensory gene, Ir41a, demonstrated an increased abundance in the antennae of older females (Fig. 4). The differential abundance of these 18 Obps, along with 3 others, appear to be a result of an increased abundance at $7 \mathrm{dpi}$ compared to the age-matched controls. The other chemosensory gene that was regulated at this age, Ir $7 u$, was down-regulated upon infection.

The age-dependent regulation of chemosensory genes was affected following $P$. falciparum infection, as $73 \%$ of those genes that were up-regulated with age (Fig. 4 column 1), were shown not to increase in abundance post-infection (Fig. 4 columns 2 and 3), resulting in a higher abundance of these transcripts in the controls compared with those at $14 \mathrm{dpi}$ (Fig. 4 column 4). The only exception to this was Ir7u, which was down-regulated at $7 \mathrm{dpi}$, exacerbating the decreased abundance observed at $14 \mathrm{dpi}$. Interestingly, when the $7 \mathrm{dpi}$ mosquitoes were compared to $14 \mathrm{dpi}$, the abundance of the above-mentioned Obps were reversed, returning to pre-infection levels (Fig. 4 column 2). 


\section{Discussion}

The stage of infection of $P$. falciparum in An. gambiae has a significant impact on vector-related behaviour, which affects malaria transmission and disease dynamics $2,5,27,28$. This study confirms that infected mosquitoes are less active than infectious females, and furthermore identifies this change in behaviour to be a result of an interaction between infection stage and age, which can be modulated by the presence of human odour and diel rhythms. Transcriptomic profiling of antennal tissue of $P$. falciparum-infected and -infectious An. gambiae identifies members of several chemosensory gene families as changing with age and infection status, suggesting a molecular mechanism, which may regulate the sensitivity of the peripheral olfactory system of the vector to human odour during the sporogonic cycle. As age is intrinsic to the infection status of the female, controlling for age provides a more accurate indication of which behaviours and molecular correlates are regulated by $P$. falciparum infection.

Host seeking in mosquitoes is dependent on age $20,21,23,29,30$ and physiological state, including infection ${ }^{3-}$ 5 . Our results demonstrated that the overall locomotor activity of uninfected females significantly reduced with age, in line with previous studies on mosquitoes ${ }^{21,23}$, further emphasising the role of age in regulating host seeking. As mosquitoes age, energy reserves decrease, resulting in older females reducing their search activities and becoming more selective to reduce their energy requirements ${ }^{31,32}$. The presence of human odour did not alter the overall locomotor activity, similar to that described by Cator et al. ${ }^{10,28}$, suggesting that the difference in the level of activity with age is independent of the presence or absence of human odour. While previous studies on the effects of Plasmodium infection on mosquito host seeking have shown a reduced activity in infected compared with infectious mosquitoes, these have not controlled for the age of the insects ${ }^{10,11}$. Our results indicate an interaction between age and infection in infected females that display a similar locomotor activity as an age-matched cohort, which is not seen in infectious individuals. When controlling for age and observing the effect of infection, infected females reduced their overall locomotor activity compared to age-matched controls in the absence of human odour, while in the presence of human odour there was no discernible difference in activity. In contrast, infectious mosquitoes increased their overall locomotor activity in the presence of human odour, but not in its absence, compared to age-matched controls. These results provide a rationale to explain why previous studies have presented opposing findings concerning the activity of oocyst-infected and sporozoite-infectious females ${ }^{10,33}$. The mechanisms underlying the changes in host-seeking and bloodfeeding behaviours are still unclear, however several hypotheses have been advanced, chief among these are that the response in infected and infectious females is a result of a general immune response, and/or a direct manipulation of the vector behaviour by the pathogen ${ }^{3,34-36}$. The increased response to human odour by infectious mosquitoes coincides with increased feeding frequency, probing rate and meal size $28,35,37,38$, and has substantial effects on malaria transmission ecology and overall disease dynamics ${ }^{36}$. 
Diel locomotor activity in mosquitoes is regulated by the age and infection status of female malaria vectors $26,39,40$. In the presence and absence of human odour, younger uninfected mosquitoes (13 days post-emergence, dpe) were more active than the older mosquitoes (19 dpe) during photophase,

scotophase and peaking at dawn ${ }^{41}$. The peak of activity for older females, on the other hand, occurred at dusk. The age-dependent change in diel locomotor activity was not modulated by the presence of human odour, which is in line with the diel patterns of the peripheral olfactory system responsiveness ${ }^{42-44}$. The shift in peak activity of older females co-occurs with an increased propensity of older females to visit nectar sources at dusk ${ }^{45}$, indicating a behavioural compartmentalisation due to an age-related reduction in energy reserves ${ }^{31,46}$. Infection with $P$. falciparum altered the diel locomotor activity in both the presence and absence of human odour, generally decreasing activity in infected and infectious females in the absence of human odour, similar to that described in An. stephensi infected with Plasmodium yoelii ${ }^{47}$. In the presence of human odour, infected and infectious females demonstrated a disrupted activity pattern associated with host seeking. In particular, infectious females shifted their odour-associated activity to scotophase and dawn, which is in line with observations of field-caught mosquitoes carrying sporozoites $^{39,48}$. By selectively increasing their activity during times of low-risk of predation and high access to human hosts, the infectious females increase the probability of a successful blood meal and pathogen transmission ${ }^{49-53}$. While the infectivity of the bite is not affected by diel rhythms ${ }^{54}$, the altered host-related activity pattern in infectious females may have explicit implications on the most effective deployment of vector control tools and thus our ability to affect malaria transmission dynamics efficiently ${ }^{36}$. Whether this is a result of an active manipulation by the pathogen or a general immune response remains to be clarified ${ }^{3,34-36,55,56}$.

Age and $P$. falciparum infection significantly affected overall antennal transcript abundance, with infection systemically down-regulating gene abundance, and differentially affecting the functional ontological class odorant binding (G0:0005549). Among the differentially abundant transcripts, antennal abundance generally increased with age across all of the major olfactory-related gene families, whereas only Obps, the most abundantly expressed transcripts of the chemosensory gene families, exhibited higher abundance in infected compared with infectious females. Odorant binding proteins are involved in binding and transporting odorants to the receptors and in the overall sensitivity of the peripheral olfactory system by providing gain control $22,57,58$. The Obps appear to play a major role in regulating the sensitivity and selectivity of the peripheral olfactory system of An. gambiae in response to aging and infection status, as $67 \%$ of the Obp transcripts reliably expressed in the antennae were age- and infectiondependently regulated. As the females age, older individuals exhibited a higher transcript abundance in two thirds of the differentially expressed classic, and all of the plus-C, classified $O b p s^{17,57}$. The other third of the differentially regulated classic Obps, along with all 13 of the two-domain $\mathrm{Obps}^{17,57}$, were expressed abundantly in $7 \mathrm{dpi}$ antennae compared with not being reliably expressed in their age-matched controls and infectious females. Bimolecular protein interaction models indicate that the most energetically favourable ligands of the reliably expressed classic Obps, including those that were differentially abundant, are distinct sets of carboxylic acids, aromatics and terpenes ${ }^{17}$, known to regulate host and 
floral seeking ${ }^{45,46}$. While no clear pattern of ligand binding was apparent associated with aging, those classic Obps increasing in abundance 7 dpi generally did not include carboxylic acids among their modelled ligands, likely resulting in a reduced sensitivity of the peripheral olfactory system to these salient human odorants by infected females ${ }^{59-61}$. This corresponds to the reduction in human odourassociated activity exhibited by infected females.

The functionally characterised differentially abundant olfactory receptors, Or11, Or39 and Ir41a, all respond to human odour constituents ${ }^{16,21,62}$. The receptor Or39 has been postulated to modulate the onset of host seeking in Anopheles coluzzii through the concerted downregulation of the receptor and the sensitivity to sulcatone of its cognate olfactory sensory neuron ${ }^{21}$. Whether the lower abundance of Or39, in conjunction with Or11, observed in the antennae of infectious compared with uninfected females underlies the observed increased locomotor activity of infectious females in the presence of human odour, remains to be investigated. The only receptor to have a higher abundance in the antennae of infectious compared to infected females, Ir47a, responds to the human spermous cyclic amine pyrrolidine $e^{16,63}$, suggesting that this receptor is involved in the increased attraction to human odour 14 dpi. The differential regulation of transcript abundance of genes modulating the chemosensory pathway in response to parasite development within the insect vector provides a mechanism by which the physiological ${ }^{14}$ and behavioural sensitivity $4,10,11,33,35$ to human odour can be modulated during the sporogonic cycle of $P$. falciparum.

Behavioural activity of An. gambiae and its expression of chemosensory-related genes are altered in an age- and $P$. falciparum stage-dependent manner. Irrespective of the mechanism regulating these changes, be it active manipulation by the parasite and/or general response to immune challenge, the differential behavioural response to human odour of females as they age or become infected significantly affects vectorial capacity and malaria transmission. Our ability to increase the resolution concerning which behavioural change is affected by age and/or infection, allows us to fine tune the epidemiological models for malaria transmission ${ }^{33}$, and integrate both factors and their interactions into the development and implementation of control measures.

\section{Methods}

\section{Ethics}

Human blood (type 0) was provided in citrate-phosphate-dextrose-adenine anti-coagulant/preservative, and serum (type $A B$ ) was obtained from the blood transfusion service (blood bank) at the Karolinska University Hospital (Solna, Sweden), in accordance with the Declaration of Helsinki, and approved by the Ethical Review Board in Stockholm (2011/850-32). The human materials were obtained anonymously from the blood bank and did not require an informed consent statement. The Swedish Work Environment Authority (Stockholm, Sweden, SU FV-2.10.2-2905-13/31-01-2022) approved the class 3 biological agent laboratory and its practices, including insectary design and equipment to work with $P$. falciparum-infected 
mosquitoes. The regulations are mainly based on the EU directive 2000/54/EC on the protection of workers from risks related to exposure to biological agents at work.

\section{Mosquito rearing and blood feeding}

Anopheles gambiae (Keele) were reared and maintained at $27 \pm 1{ }^{\circ} \mathrm{C}, 80 \pm 1 \%$ relative humidity and a photoperiod of $12 \mathrm{~h}$ light: $12 \mathrm{~h}$ dark cycle, $11 \mathrm{~h}$ full light of $\sim 300 \mathrm{~lx}$ and $11 \mathrm{~h}$ darkness, separated by $1 \mathrm{~h}$ dawn and dusk transitions, respectively. The Keele colony was established at Keele University as a result of balanced interbreeding of four An. gambiae s.I. strains: G3 from MacCarthy Island, the Gambia; and three Tanzanian strains, ZAN U from Zanzibar, Ifakara strain from Njage, and KIL from Marangu ${ }^{64}$, and set up at Stockholm University in 2014. For experiments, pupae (ca. 350) were collected and transferred to cages (custom made: $30 \mathrm{~cm} \times 20 \mathrm{~cm} \times 20 \mathrm{~cm}$ ) to emerge as adults, which were fed ad libitum on $5 \%$ glucose and $0.05 \%$ para-amino benzoic acid (PABA, Sigma-Aldrich, Steinheim, Germany). Females (5 dpe) were provided with a meal of cultured red blood cells (RBCs) through glass membrane-feeders connected to a custom-built heating system kept at $37^{\circ} \mathrm{C}$. The RBCs were washed with Roswell Park Memorial Institute (RPMI-1640) medium (Gibco ${ }^{\mathrm{TM}}$, ThermoFisher Scientific, Drieich, Germany), and stored in RPMI1640 at $50 \%$ haematocrit at $4{ }^{\circ} \mathrm{C}$ prior to use.

\section{Parasite culture and mosquito infection}

The strain of $P$. falciparum used in this study, $\mathrm{NF}_{54}-\mathrm{SU}$, was initially isolated in the Netherlands and donated by Klavs Berzins. In vivo culturing of $P$. falciparum $\mathrm{NF}_{54}-\mathrm{SU}$ followed the standard protocol ${ }^{65}$. In short, $P$. falciparum $\mathrm{NF}_{54}$-SU was cultured using washed $\mathrm{RBCs}$ ( $5 \%$ haematocrit) from type-0 blood donors, and $10 \%$ serum mixed with RPMI-1640 medium (Gibco ${ }^{\mathrm{TM}}$, ThermoFisher Scientific), supplemented with $5.94 \mathrm{~g} \mathrm{l}^{-1}$ HEPES buffer (Sigma Aldrich), $2.1 \mathrm{~g} \mathrm{l}^{-1}$ sodium hydrogen carbonate (Sigma Aldrich), and $0.05 \mathrm{~g} \mathrm{l}^{-1}$ hypoxanthine (Sigma Aldrich). The cultures were kept in controlled conditions under gas $\left(1 \% \mathrm{O}_{2}\right.$, $3 \% \mathrm{CO}_{2}$ and $96 \% \mathrm{~N}_{2}$ ) at $37^{\circ} \mathrm{C}$. Plasmodium falciparum gametocyte cultures were set up at $0.5-0.7 \%$ parasitaemia, $6 \%$ haematocrit in RPMI-1640 medium. On the day of experimental infection, uninfected RBCs were supplemented with $P$. falciparum gametocytes to a final gametocytaemia of ca. $0.7 \%$, which generates a high infection prevalence $(>50 \%)$ in An. gambiae s.s ${ }^{65}$. For each infectious meal, mixtures of gametocytes from 14- and 17-day gametocyte cultures were used. Twenty-eight groups of ca. 100 mosquitoes were fed on mature gametocyte-infected RBCs or on uninfected washed RBCs using a membrane feeder. All age-matched control females ( 7 and 14 days control ; 7 and $14 \mathrm{dc}$ ) were handled in the same manner as the infected and infectious cohorts, including access to mates, blood meals and oviposition substrates.

\section{Mosquito odour-mediated locomotor activity}

For recording the behavioural activity of the mosquitoes, DAM2 Drosophila activity monitors (TriKinetics Inc., Waltham, MA, USA) were used. Two hours prior to the experiments, mosquitoes were placed individually in glass tubes (65 mm $\otimes 7 \mathrm{~mm}$ ), using an aspirator, for pre-conditioning. Cotton plugs 
saturated with $5 \%$ glucose with $0.05 \%$ PABA (as above) were placed at one end of the glass tubes to provide ad libitum access to sustenance. Human odour was supplied by a previously worn cotton sock (ca. $24 \mathrm{~h}$ ) placed inside a $200 \mathrm{ml}$ glass wash bottle (Sigma Aldrich). The odour was introduced, via Teflon tubing, into the glass tubes through a MAN2 gas distribution manifold (TriKinetics Inc.). A clean unworn sock was similarly used as a control. Each experiment was replicated three times with infected and infectious mosquitoes assessed in parallel with age-matched controls, for a total of 384 mosquitoes assayed ( 96 per condition). Behavioural experiments were conducted in the class 3 biological agent laboratory (BSL3) within a climate chamber kept at $27 \pm 1{ }^{\circ} \mathrm{C}, 70 \%$ relative humidity with a photoperiod of $11 \mathrm{~h}$ light: $11 \mathrm{~h}$ dark with 60 min of both dawn and dusk lighting conditions. Both assays were conducted over $24 \mathrm{~h}$, with activity data recorded every $30 \mathrm{~s}$.

The influence of age, the presence and absence of human odour, and the effect of the sporogonic stages of $P$. falciparum on the An. gambiae ( 7 and 14 days post infection; 7 and $14 \mathrm{dpi}$ ), diel locomotor activity was analysed using general linear mixed models (GLMM), in which the effect of replication (experimental blocks) and the weighting of multiple replications (random variables) were taken into account. For all results, the significance of the best maximal model containing all explanatory effects was evaluated by using likelihood ratio test. All analyses were performed using R ( $R$ Core team ${ }^{66}: \mathrm{R}$ v.3.2.3 and RStudio 1.1.463 ${ }^{67}$ ). The $b$-estimated values of locomotor activity were estimated in all final significant models (GLMM: Imer function; in Fig 1b: The mixed model was build based on the effect of age as a main fixed variable combined with the random effect of the other variables; Fig 1c: The model was demonstrated the effect of the main fixed variable of infection including the rest of the variables as random variables; Fig $1 \mathrm{~d}$ : The mixed model was estimated the effect of all four main fixed variables including age, infection, time, human odour, as well as the random effect of the experimental replication combined with the interactions among all these variables). In all analyses, all of the data met the assumptions of the test for normality and error homogeneity. Backward elimination was used for sequential removal of nonsignificant variables, to obtain the minimal statistically significant model. All graphical visualisations and statistical analyses were preformed using GraphPad Prism ${ }^{68}$ and R (R Core team ${ }^{66}: \mathrm{R}$ v.3.2.3 and RStudio $\left.1.1 .463^{67}\right)$. The precise levels of significance are presented in the results section, except the universally accepted $p<0.001$.

\section{Transcriptome analyses}

Pairs of antennae from infected, infectious and age-matched control mosquitoes were collected from individual females following flash freezing on dry ice for $<30 \mathrm{~s}$, and placed into 24 -well culture plates (ThermoFisher Scientific) in RNAlater (500 $\mu$ l; Thermo Fisher Scientific). Individual carcasses (bodies missing antennae) of infected and infectious mosquitoes were also collected for subsequent qPCR analysis, to determine the parasite abundance of individual females (see below). Following the quantification of the parasite load, the antennae of successfully infected individuals were pooled into tubes of 150 pairs of antennae, according to the level of infection, and stored at $-80^{\circ} \mathrm{C}$. Four replicates of each condition were collected. RNA libraries were constructed using a TruSeq RNA Library Prep Kit (Illumina, Berlin, Germany) from total RNA from the pooled antennae following extraction and DNase I 
digestion (RNeasy Mini kit, Qiagen, Hilden, Germany). Quality and quantity control of total RNA aliquots was performed using a Nanodrop spectrophotometer (Thermo Fisher Scientific), an Agilent 2100 Bioanalyser (Santa Clara, CA, USA) and a Qubit 2.0 Fluorometer (Thermo Fisher Scientific), before sending to BGI Genomics (MGI Tech Co., Ltd., China) for Illumina paired-end indexed sequencing, using the Illumina HiSeq2000.

\section{RNA-Seq and differential expression analyses}

High-quality reads were determined using the following criteria: low quality reads were clipped from the start and end of each read using a sliding window and reads shorter than $40 \mathrm{nt}$ were removed. The cleaned reads were mapped using CLC Genomics Workbench version 11 (https://digitalinsights.qiagen.com) to the An. gambiae genome Agam4 with reference to the Agam4.10 gene set (www.vectorbase.org). On average $94.11 \%$ of all reads mapped to the genome. Transcript abundance was reported as transcript per million (TPM) with a threshold level of abundance above 1 TPM. Differential transcript abundance was determined using the $\beta$-binomial general linear model algorithms in CLC Genomics Workbench 11. To control for false discovery rate (FDR), the BenjaminiHochberg correction was applied ${ }^{69}$. This analysis generated weighted fold changes (FC) and FDRcorrected $p$-values that were used to detect differential expression. Significantly differential gene abundance was determined based on a FC $\geq 2$ and a FDR-corrected $p$-value $<0.05$.

\section{Quantitative real-time polymerase chain reaction analysis}

Quantitative real-time polymerase chain reaction(qPCR) was used to estimate the abundance of $P$. falciparum in individual mosquitoes subjected to an infectious meal at 7 and $14 \mathrm{dpi}$, and used for both behavioural and transcriptomic analyses, allowing quantification of the numbers of parasite genomes. In all analyses, a single body of an uninfected female mosquito was used as a negative control. Reactions were performed on a Roche Light Cycler using SYBR Green (Roche, Mannheim, Germany). DNA standards containing known numbers of $P$. falciparum parasites were produced from asexual cultures, and used to generate standard curves of $P$. falciparum. A similar statistical approach, as described for the behavioural analyses, was used to test for variation in the number of parasites (oocyst load and total number of sporozoites per whole mosquito body $)^{70}$. Given the highly over-dispersed nature of parasite abundance data, a negative binomial distribution was assumed in these analyses (GLMM: glmmADMB, nlme package; $\mathrm{R}$ Core team ${ }^{66}$ : R v.3.2.3 and RStudio 1.1.463 $\left.{ }^{66}\right)$. A backwards elimination approach was used to test for the significance of all fixed effects and interactions, while controlling for random variation due to replicate, as for the analyses of oocyst and sporozoite prevalence.

\section{Declarations}

\section{Data availability}

All data supporting the findings of this study are available within the article and its Supplementary Information files. The sequencing data are available at the GEO database (BioProject ID PRJNA756244), 
and are available from the corresponding authors upon request.

\section{Acknowledgements}

We thank the Swedish Research Council for funding to RI (VR/2014-3331) and SNE (VR/2017-01229; VR/2017-05543 UFNW). We extend our thanks to Jeansson's Stiftelser (SJ-2018) for the valuable support for setting up the essential equipment of the BSL3 laboratory. We highly appreciate the donation of the $\mathrm{NF}_{54}-\mathrm{SU}$ strain of $P$. falciparum by Prof. Klavs Berzins.

\section{Author Contributions}

$\mathrm{RI}$ and SNE conceived the study and designed the experiments. SNE and MH cultured the parasites, performed the mosquito infections as well as conducting the behavioural analyses. All statistical and modelling analyses for behavioural assays were carried out by $\mathrm{MH}$ and SNE for the deep analysing of the mixed model data. LRC, RM and SNE dissected the antenna and prepared RNA for Illumina sequencing. $\mathrm{RI}, \mathrm{SRH}$ and SNE designed the transcriptome study, and MH and SRH performed the analyses in consultation SNE. MH, RI, SRH and SNE wrote the manuscript. All authors read and approved the manuscript.

\section{Competing interests}

The authors declare no competing interests. S. Noushin Emami is a co-founder of Molecular Attraction $A B$ and there are no commercial aspects or financial gain to this company resulting from this study.

\section{Materials and correspondence}

Correspondence and materials requests should be directed to S. Noushin Emami, noushin.emami@su.se; and Rickard Ignell, rickard.ignell@slu.se

\section{References}

1. De Moraes, C. M. et al. Malaria-induced changes in host odors enhance mosquito attraction. Proc Natl Acad Sci U S A 111, 11079-11084, doi:10.1073/pnas.1405617111 (2014).

2. Emami, S. N. et al. A key malaria metabolite modulates vector blood seeking, feeding, and susceptibility to infection. Science 10, doi:10.1126/science.aah4563 (2017).

3. Hurd, H. Manipulation of medically important insect vectors by their parasites. Annu Rev Entomol 48, 141-161, doi:10.1146/annurev.ento.48.091801.112722 (2003).

4. Lefevre, T. \& Thomas, F. Behind the scene, something else is pulling the strings: emphasizing parasitic manipulation in vector-borne diseases. Infect Genet Evo/ 8, 504-519, doi:10.1016/j.meegid.2007.05.008 (2008).

5. Tallon, A. K. et al. Dengue infection modulates locomotion and host seeking in Aedes aegypti. PLoS Negl Trop Dis 14, e0008531, doi:10.1371/journal.pntd.0008531 (2020). 
6. Botto-Mahan, C., Cattan, P. E. \& Medel, R. Chagas disease parasite induces behavioural changes in the kissing bug Mepraia spinolai. Acta Tropica 98, 219-223, doi:10.1016/j.actatropica.2006.05.005 (2006).

7. Lefevre, T. et al. Malaria Plasmodium agent induces alteration in the head proteome of their Anopheles mosquito host. Proteomics 7, 1908-1915, doi:10.1002/pmic.200601021 (2007).

8. Biron, D. G. et al. Behavioural manipulation in a grasshopper harbouring hairworm: a proteomics approach. Proceedings of the Royal Society B-Biological Sciences 272, 2117-2126, doi: 10.1098/rspb.2005.3213 (2005).

9. Beier, J. C. Malaria parasite development in mosquitoes. Annual Review of Entomology 43, 519-543, doi: 10.1146/annurev.ento.43.1.519 (1998).

10. Cator, L. J. et al. 'Manipulation' without the parasite: altered feeding behaviour of mosquitoes is not dependent on infection with malaria parasites. Proc Biol Sci 280, 20130711, doi:10.1098/rspb.2013.0711 (2013).

11. Smallegange, R. C. et al. Malaria infected mosquitoes express enhanced attraction to human odor. Plos One 8, e63602, doi:10.1371/journal.pone.0063602 (2013).

12. Cornet, S., Nicot, A., Rivero, A. \& Gandon, S. Avian malaria alters the dynamics of blood feeding in Culex pipiens mosquitoes. Malar J 18, 82, doi:10.1186/s12936-019-2690-5 (2019).

13. Padilha, K. P. et al. Zika infection decreases Aedes aegypti locomotor activity but does not influence egg production or viability. Mem Inst Oswaldo Cruz 113, e180290, doi:10.1590/0074-02760180290 (2018).

14. Stanczyk, N. M. et al. Species-specific alterations in Anopheles mosquito olfactory responses caused by Plasmodium infection. Sci Rep 9, 3396, doi:10.1038/s41598-019-40074-y (2019).

15. Lu, T. et al. Odor coding in the maxillary palp of the malaria vector mosquito Anopheles gambiae. Curr Biol 17, 1533-1544, doi:10.1016/j.cub.2007.07.062 (2007).

16. Pitts, R. J., Derryberry, S. L., Zhang, Z. \& Zwiebel, L. J. Variant ionotropic receptors in the malaria vector mosquito Anopheles gambiae tuned to amines and carboxylic acids. Sci Rep 7, 40297, doi:10.1038/srep40297 (2017).

17. Manoharan, M. et al. Comparative genomics of odorant binding proteins in Anopheles gambiae, Aedes aegypti, and Culex quinquefasciatus. Genome Biol Evol 5, 163-180, doi:10.1093/gbe/evs131 (2013).

18. Bohbot, J. D. et al. Conservation of indole responsive odorant receptors in mosquitoes reveals an ancient olfactory trait. Chem Senses 36, 149-160, doi:10.1093/chemse/bjq105 (2011).

19. Erdelyan, C. N., Mahood, T. H., Bader, T. S. \& Whyard, S. Functional validation of the carbon dioxide receptor genes in Aedes aegypti mosquitoes using RNA interference. Insect Mol Bio/ 21, 119-127, doi:10.1111/j.1365-2583.2011.01120.x (2012).

20. Omondi, B. A., Majeed, S. \& Ignell, R. Functional development of carbon dioxide detection in the maxillary palp of Anopheles gambiae. J Exp Bio/ 218, 2482-2488, doi:10.1242/jeb.116798 (2015). 
21. Omondi, A. B., Ghaninia, M., Dawit, M., Svensson, T. \& Ignell, R. Age-dependent regulation of host seeking in Anopheles coluzzii. Sci Rep 9, 9699, doi:10.1038/s41598-019-46220-w (2019).

22. Rinker, D. C. et al. Blood meal-induced changes to antennal transcriptome profiles reveal shifts in odor sensitivities in Anopheles gambiae. Proc Natl Acad Sci U S A 110, 8260-8265, doi:10.1073/pnas.1302562110 (2013).

23. Tallon, A. K., Hill, S. R. \& Ignell, R. Sex and age modulate antennal chemosensory-related genes linked to the onset of host seeking in the yellow-fever mosquito, Aedes aegypti. Sci Rep 9, 43, doi:10.1038/s41598-018-36550-6 (2019).

24. Hill, S. R. \& Ignell, R. Modulation of odour-guided behaviour in mosquitoes. Cell and Tissue Research 383, 195-206, doi:10.1007/s00441-020-03368-6 (2021).

25. Hill, S. R., Ghaninia, M. \& Ignell, R. Blood meal induced regulation of gene expression in the maxillary palps, a chemosensory organ of the mosquito Aedes aegypti. Front Ecol Evol 7, doi:ARTN 33610.3389/fevo.2019.00336 (2019).

26. Jones, M. D. R. \& Gubbins, S. J. Changes in the circadian flight activity of the mosquito Anopheles gambiae in relation to insemination, feeding and oviposition. Physiol Entomo/3, 213-220, doi: 10.1111/j.1365-3032.1978.tb00151.x (1978).

27. Koella, J. C., Sorensen, F. L. \& Anderson, R. A. The malaria parasite, Plasmodium falciparum, increases the frequency of multiple feeding of its mosquito vector, Anopheles gambiae. Proc Biol Sci 265, 763-768, doi:10.1098/rspb.1998.0358 (1998).

28. Anderson, R. A., Koella, J. C. \& Hurd, H. The effect of Plasmodium yoelii nigeriensis infection on the feeding persistence of Anopheles stephensi Liston throughout the sporogonic cycle. Proceedings of the Royal Society B-Biological Sciences 266, 1729-1733, doi:10.1098/rspb.1999.0839 (1999).

29. Bohbot, J. D., Durand, N. F., Vinyard, B. T. \& Dickens, J. C. Functional development of the octenol response in Aedes aegypti. Front Physio/ 4, 39, doi:10.3389/fphys.2013.00039 (2013).

30. Foster, W. A. \& Takken, W. Nectar-related vs. human-related volatiles: behavioural response and choice by female and male Anopheles gambiae (Diptera: Culicidae) between emergence and first feeding. Bull Entomol Res 94, 145-157, doi:10.1079/ber2003288 (2004).

31. Rowley, W. A. \& Graham, C. L. The effect of age on the flight performance of female aedes aegypti mosquitos. J Insect Physiol 14, 719-728, doi:10.1016/0022-1910(68)90230-8 (1968).

32. Nayar, J. K. \& Sauerman, D. M., Jr. A comparative study of flight performance and fuel utilization as a function of age in females of Florida mosquitoes. $J$ Insect Physio/ 19, 1977-1988, doi:10.1016/00221910(73)90192-3 (1973).

33. Vantaux, A. et al. Host-seeking behaviors of mosquitoes experimentally infected with sympatric field isolates of the human malaria parasite Plasmodium falciparum: no evidence for host manipulation. Front Ecol Evol 3, doi:10.3389/fevo.2015.00086 (2015).

34. Cator, L. J. et al. Immune response and insulin signalling alter mosquito feeding behaviour to enhance malaria transmission potential. Sci Rep 5, 11947, doi:10.1038/srep11947 (2015). 
35. Koella, J. C., Rieu, L. \& Paul, R. E. L. Stage-specific manipulation of a mosquito's host-seeking behavior by the malaria parasite Plasmodium gallinaceum. Behavioral Ecology 13, 816-820, doi:10.1093/beheco/13.6.816 (2002).

36. Cator, L. J., Lynch, P. A., Read, A. F. \& Thomas, M. B. Do malaria parasites manipulate mosquitoes? Trends in Parasitology 28, 466-470, doi:10.1016/J.Pt.2012.08.004 (2012).

37. Rossignol, P. A., Ribeiro, J. M. \& Spielman, A. Increased intradermal probing time in sporozoiteinfected mosquitoes. Am J Trop Med Hyg 33, 17-20, doi: 10.4269/ajtmh.1984.33.17 (1984).

38. Rossignol, P. A., Ribeiro, J. M. \& Spielman, A. Increased biting rate and reduced fertility in sporozoiteinfected mosquitoes. Am J Trop Med Hyg 35, 277-279, doi:10.4269/ajtmh.1986.35.277 (1986).

39. Gillies, M. T. Age-groups and the biting cycle in Anopheles gambiae. A preliminary investigation $B$ Entomol Res 48, 553-559, doi:10.1017/S0007485300002728 (1957).

40. Bockarie, M. J. \& Dagoro, H. Are insecticide-treated bednets more protective against Plasmodium falciparum than Plasmodium vivax-infected mosquitoes? Malar J 5, 15, doi:10.1186/1475-2875-5-15 (2006).

41. Lindsay, S. W., Shenton, F. C., Snow, R. W. \& Greenwood, B. M. Responses of Anopheles gambiae complex mosquitoes to the use of untreated bednets in The Gambia. Med Vet Entomo/3, 253-262, doi:10.1111/j.1365-2915.1989.tb00225.x (1989).

42. Rund, S. S. et al. Daily rhythms in antennal protein and olfactory sensitivity in the malaria mosquito Anopheles gambiae. Sci Rep 3, 2494, doi:10.1038/srep02494 (2013).

43. Eilerts, D. F., VanderGiessen, M., Bose, E. A., Broxton, K. \& Vinauger, C. Odor-specific daily rhythms in the olfactory sensitivity and behavior of Aedes aegypti Mosquitoes. Insects $\mathbf{9}$, doi:10.3390/insects9040147 (2018).

44. Tanoue, S., Krishnan, P., Krishnan, B., Dryer, S. E. \& Hardin, P. E. Circadian clocks in antennal neurons are necessary and sufficient for olfaction rhythms in Drosophila. Curr Bio/ 14, 638-649, doi:10.1016/j.cub.2004.04.009 (2004).

45. Gary, R. E., Jr. \& Foster, W. A. Diel timing and frequency of sugar feeding in the mosquito Anopheles gambiae, depending on sex, gonotrophic state and resource availability. Med Vet Entomo/ 20, 308316, doi:10.1111/j.1365-2915.2006.00638.x (2006).

46. Foster, W. A. Mosquito sugar feeding and reproductive energetics. Annu Rev Entomol 40, 443-474, doi:10.1146/annurev.en.40.010195.002303 (1995).

47. Rowland, M. Changes in the circadian flight activity of the mosquito Anopheles-Stephensi Associated with Insemination, blood-feeding, oviposition and nocturnal Light-Intensity. Physiol Entomo/ 14, 7784, doi:DOI 10.1111/j.1365-3032.1989.tb00939.x (1989).

48. Djenontin, A., Bouraima, A., Soares, C., Egbinola, S. \& Cottrell, G. Human biting rhythm of Anopheles gambiae Giles, 1902 (Diptera: Culicidae) and sleeping behaviour of pregnant women in a lagoon area in Southern Benin. BMC Res Notes 14, 200, doi:10.1186/s13104-021-05615-7 (2021).

49. O'Donnell, A. J., Rund, S. S. C. \& Reece, S. E. Time-of-day of blood-feeding: effects on mosquito life history and malaria transmission. Parasit Vectors 12, 301, doi:10.1186/s13071-019-3513-9 (2019). 
50. Takken, W., van Loon, J. J. \& Adam, W. Inhibition of host-seeking response and olfactory responsiveness in Anopheles gambiae following blood feeding. J Insect Physio/ 47, 303-310, (2001).

51. Reece, S. E., Prior, K. F. \& Mideo, N. The Life and Times of Parasites: Rhythms in Strategies for Withinhost Survival and Between-host Transmission. J Biol Rhythms 32, 516-533, doi:10.1177/0748730417718904 (2017).

52. Martinez-Bakker, M. \& Helm, B. The influence of biological rhythms on host-parasite interactions. Trends Ecol Evol 30, 314-326, doi:10.1016/j.tree.2015.03.012 (2015).

53. Mideo, N., Reece, S. E., Smith, A. L. \& Metcalf, C. J. The Cinderella syndrome: why do malaria-infected cells burst at midnight? Trends Parasito/ 29, 10-16, doi:10.1016/j.pt.2012.10.006 (2013).

54. Githeko, A. K. et al. Confirmation that Plasmodium falciparum has aperiodic infectivity to Anopheles gambiae. Med Vet Entomo/ 7, 373-376, doi: 10.1111/j.1365-2915.1993.tb00708.x (1993).

55. Poulin, R. The evolution of parasite manipulation of host behavior - a theoretical-analysis. Parasitology 109, S109-S118, doi: 10.1017/S0031182000085127 (1994).

56. Emami, S. N., Hajkazemian, M. \& Mozuraitis, R. Can Plasmodium's tricks for enhancing its transmission be turned against the parasite? New hopes for vector control. Pathog Glob Health 113, 325-335, doi:10.1080/20477724.2019.1703398 (2019).

57. Li, Z. X., Pickett, J. A., Field, L. M. \& Zhou, J. J. Identification and expression of odorant-binding proteins of the malaria-carrying mosquitoes Anopheles gambiae and Anopheles arabiensis. Arch Insect Biochem Physio/ 58, 175-189, doi:10.1002/arch.20047 (2005).

58. Larter, N. K., Sun, J. S. \& Carlson, J. R. Organization and function of Drosophila odorant binding proteins. Elife 5, doi:10.7554/eLife.20242 (2016).

59. Acree, F., Jr., Turner, R. B., Gouck, H. K., Beroza, M. \& Smith, N. L-Lactic acid: a mosquito attractant isolated from humans. Science 161, 1346-1347, doi:10.1126/science.161.3848.1346 (1968).

60. Raji, J. I. et al. Aedes aegypti mosquitoes detect acidic volatiles found in human odor using the IR8a Pathway. Curr Bio/ 29, 1253-1262 e1257, doi:10.1016/j.cub.2019.02.045 (2019).

61. Cork, A. \& Park, K. C. Identification of electrophysiologically-active compounds for the malaria mosquito, Anopheles gambiae, in human sweat extracts. Med Vet Entomo/ 10, 269-276, doi:10.1111/j.1365-2915.1996.tb00742.x (1996).

62. Carey, A. F., Wang, G., Su, C. Y., Zwiebel, L. J. \& Carlson, J. R. Odorant reception in the malaria mosquito Anopheles gambiae. Nature 464, 66-71, doi:10.1038/nature08834 (2010).

63. Amoore, J. E., Forrester, L. J. \& Buttery, R. G. Specific Anosmia to 1-Pyrroline: The Spermous Primary Odor. J Chem Ecol 1, 299-310, doi:10.1007/Bf00988831 (1975).

64. Hurd, H., Taylor, P. J., Adams, D., Underhill, A. \& Eggleston, P. Evaluating the costs of mosquito resistance to malaria parasites. Evolution 59, 2560-2572, doi: 10.1554/05-211.1 (2005).

65. Carter, R., Ranford-Cartwright, L. \& Alano, P. The culture and preparation of gametocytes of Plasmodium falciparum for immunochemical, molecular, and mosquito infectivity studies. Methods Mol Biol 21, 67-88, doi:10.1385/0-89603-239-6:67 (1993). 
66. R Core Team. R: a language and environment for statistical computing, <https://www.R-project.org/> (2020).

67. RStudio-Team. RStudio: Integrated Development for R. . RStudio, doi: http://www.rstudio.com/ (2021).

68. GraphPad Prism. GraphPad Software version 6.04, <http://www.graphpad.com/> (2020).

69. Trapnell, C. et al. Differential analysis of gene regulation at transcript resolution with RNA-seq. Nat Biotechno/ 31, 46-53, doi:10.1038/nbt.2450 (2013).

70. Emami, S. N., Ranford-Cartwright, L. C. \& Ferguson, H. M. The transmission potential of malariainfected mosquitoes (An.gambiaeKeele, An.arabiensis-Ifakara) is altered by the vertebrate blood type they consume during parasite development. Sci Rep7, 40520, doi:10.1038/srep40520 (2017).

\section{Figures}

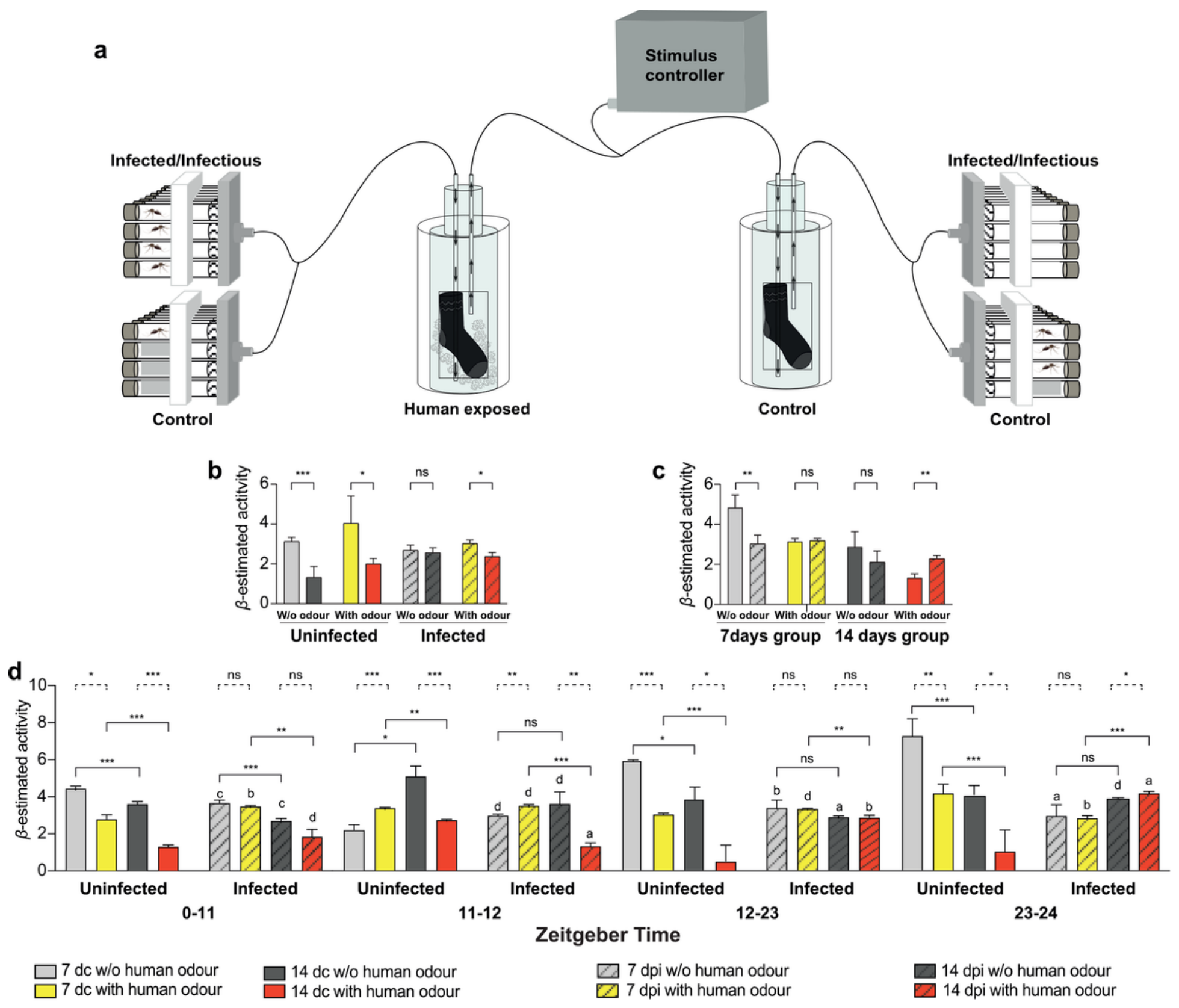




\section{Figure 1}

Plasmodium falciparum infection stage differentially affects mosquito locomotor activity. Schematic design of the locomotor assay (a) used to assess the activity of female Anopheles gambiae, 7 days postinfection (dpi) and 14 dpi with P. falciparum compared to age-matched uninfected females (days control, dc), in the presence (with human odour) or absence of human odour (w/o human odour). Individual locomotor profiles $(n=384)$, assessed for variation using a redundancy analysis, demonstrated a differential effect of age (The mixed model was build based on the effect of age as a main fixed variable combined with the random effect of the other variables; $\beta$-Imer $\pm S E=$ age + experimental replication $\mid 1 ; b)$ and infection (The model was demonstrated the effect of the main fixed variable of infection including the rest of the variables as random variables; $\beta$-Imer $\pm S E=$ infection + experimental replication|1; $c$ ) on locomotor activity in the presence and absence of human odour. (d) Diel locomotor activity profiles of 7 and 14 dpi females, along with age-matched controls, demonstrated effects of both age and infection in the presence and absence of human odour during informative activity periods (photophase, Zeitgeber Time (ZT) 0-11; dusk, ZT 11-12; scotophase, ZT 12-23; and dawn, ZT 23-34) over a 24 hour period (The mixed model was estimated the effect of all four main fixed variables including age, infection, time, human odour, as well as the random effect of the experimental replication combined with the interactions among all these variables; $\beta$-Imer $\pm \mathrm{SE}=$ age + infection + time + human odour + experimental replication|1). Significant effects were determined using GLMM models (Imer). Bars represented by $\beta$ estimation generated by the mixed model \pm SE $(\beta$-Imer $\pm S E)$; asterisks denote significant differences $\left({ }^{*} p<\right.$ $0.05 ;{ }^{* \star} p<0.01 ;{ }^{* \star} \mathrm{p}<0.001$; ns, non-significant). Letters indicate the pairwise comparisons between infected and uninfected groups; $a=p<0.001, b=p<0.01, c=p<0.05, d=$ non-significant). 

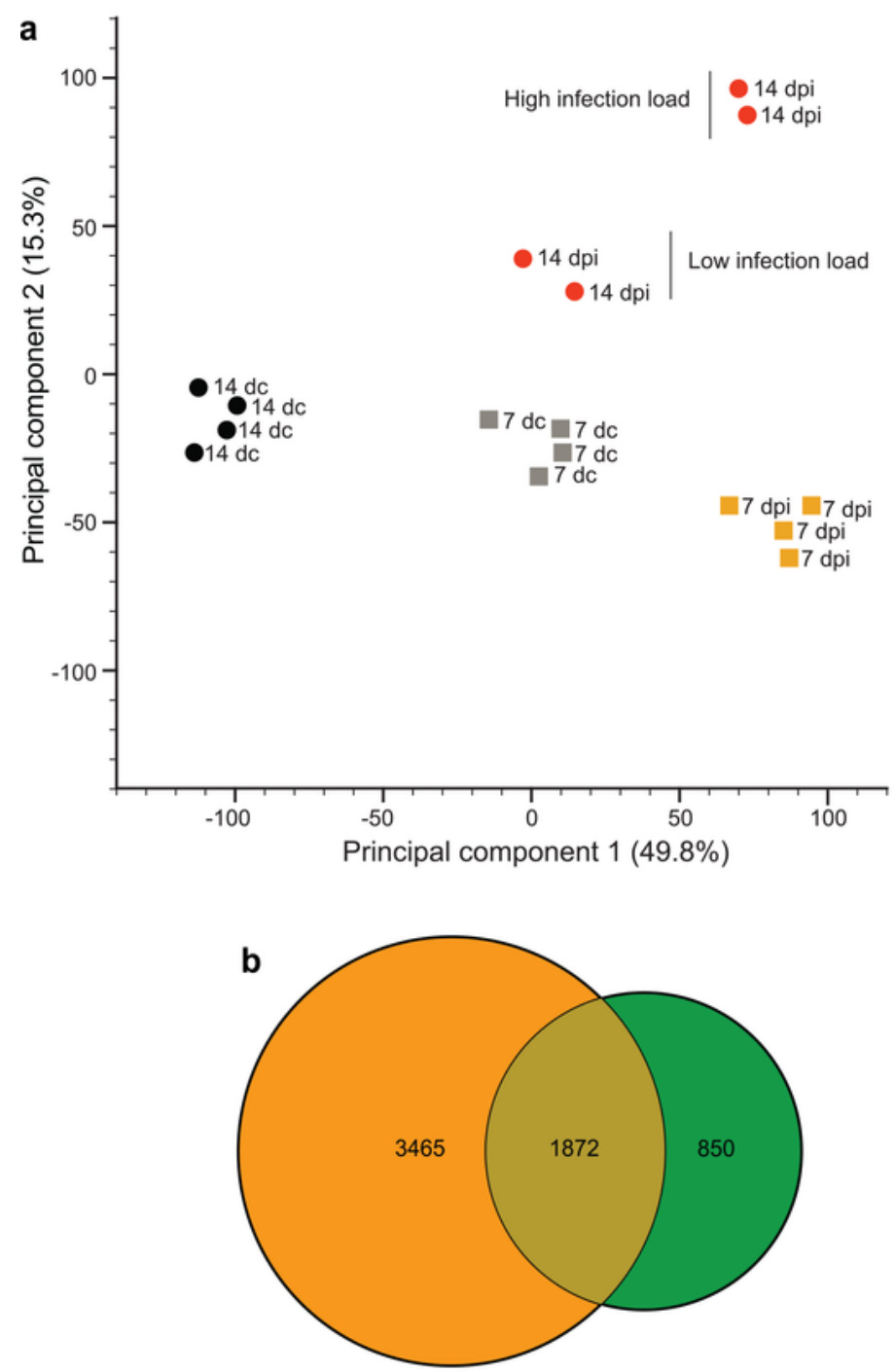

14 dpi vs. 14 dc 7 dpi vs. 7 dc

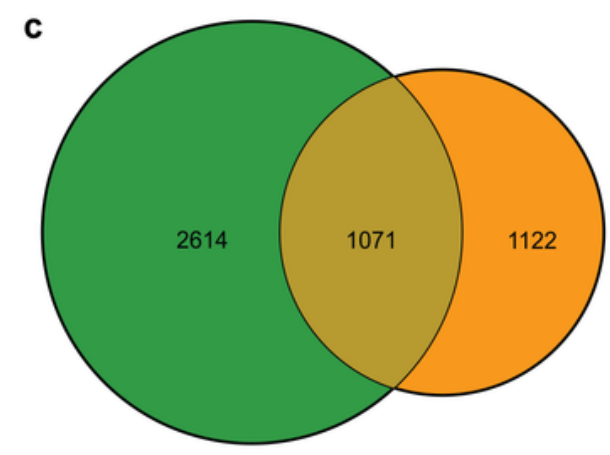

14 dc vs. 7 dc 14 dpi vs. 7 dpi

\section{Figure 2}

Plasmodium falciparum infection stage affects antennal transcript abundance in Anopheles gambiae . Principal component analysis (a) of the overall transcript abundance in the antennae of infected (7 days post-infection, dpi) and infectious ( $14 \mathrm{dpi}$ ) mosquitoes compared to age-matched uninfected mosquitoes ( 7 days control, dc and $14 \mathrm{dc}$ ). Plasmodium falciparum infection load is indicated (see Supplementary Figure 1). Proportional Venn diagrams (b-c) depicting pairwise comparisons among the antennal 
transcriptomes of infected, infectious, and age-matched uninfected females. Overlapping regions represent the subsets of transcripts that are shared between the different conditions. Significant differences in transcript abundance were determined as a fold change of greater than 1.5 , and an FDRcorrected p-value $<0.05$.

a

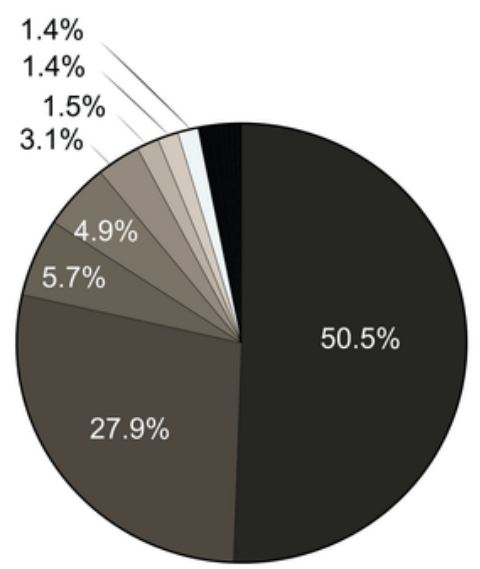

$14 \mathrm{dc}$ vs $7 \mathrm{dc}$

b

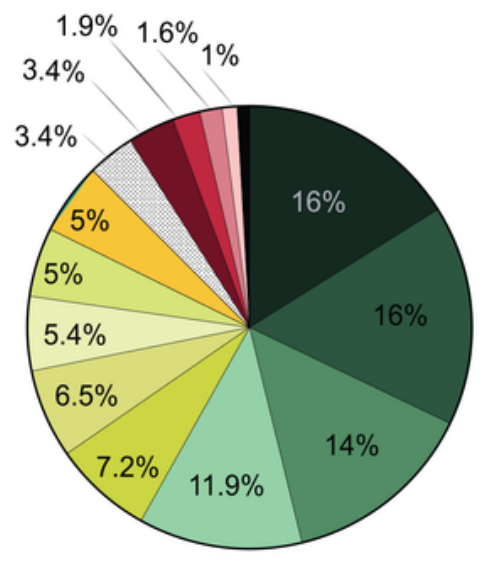

7 dpi vs $7 \mathrm{dc}$

Heterocyclic compound binding Organic cyclic compound binding lon binding

Transferase activity

Small molecule binding

Hydrolase activity

Carbohydrate derivative binding

Drug binding

Transporter activity

Oxidoreductase activity

Protein binding

Cofactor binding

Molecular function regulatory

Odorant binding

Metalloenzyme regulator activity
Structural constituent of cuticle

Enzyme inhibitor activity

Metal cluster binding

Neurotransmitter binding

$\square$ Peptide regulator activity

$\square$ Channel inhibitor activity

$\square$ Ras guanyl-nucleotide exchange factor activity

$\square$ Carbohydrate binding

ather

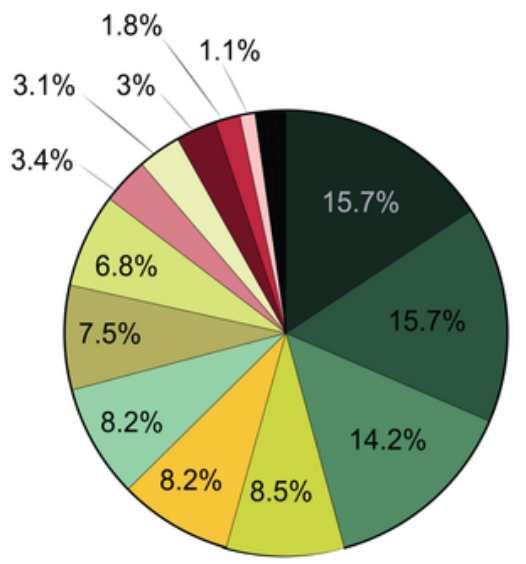

7 dpi vs 14 dpi

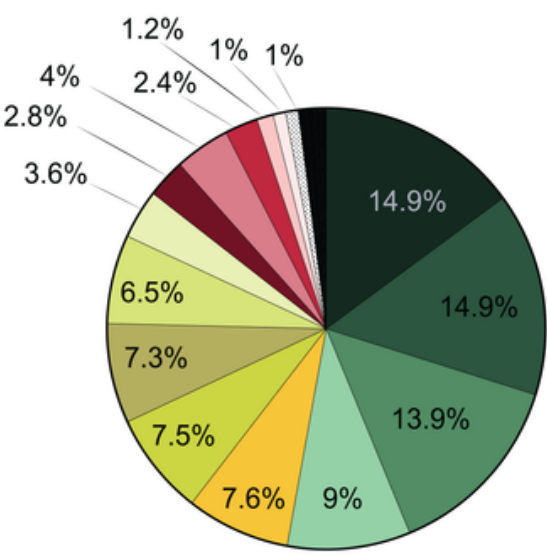

$14 \mathrm{dpi}$ vs $14 \mathrm{dc}$

Figure 3 
Plasmodium falciparum infection stage affects the functional ontology of differentially abundant antennal transcripts. A level 3 gene ontology analysis of molecular function of Anopheles gambiae antennal transcriptomes comparing those of 13 and 19 days post-emergence females ( 7 days control, dc and $14 \mathrm{dc}$, respectively) (a), as well as those of 7 and 14 days post-infection (dpi) females along with age-matched controls (b). Gene ontology classes that were included comprised greater than $1 \%$ of the total number of differentially abundant genes identified. Those with less than $1 \%$ are grouped together in "other".

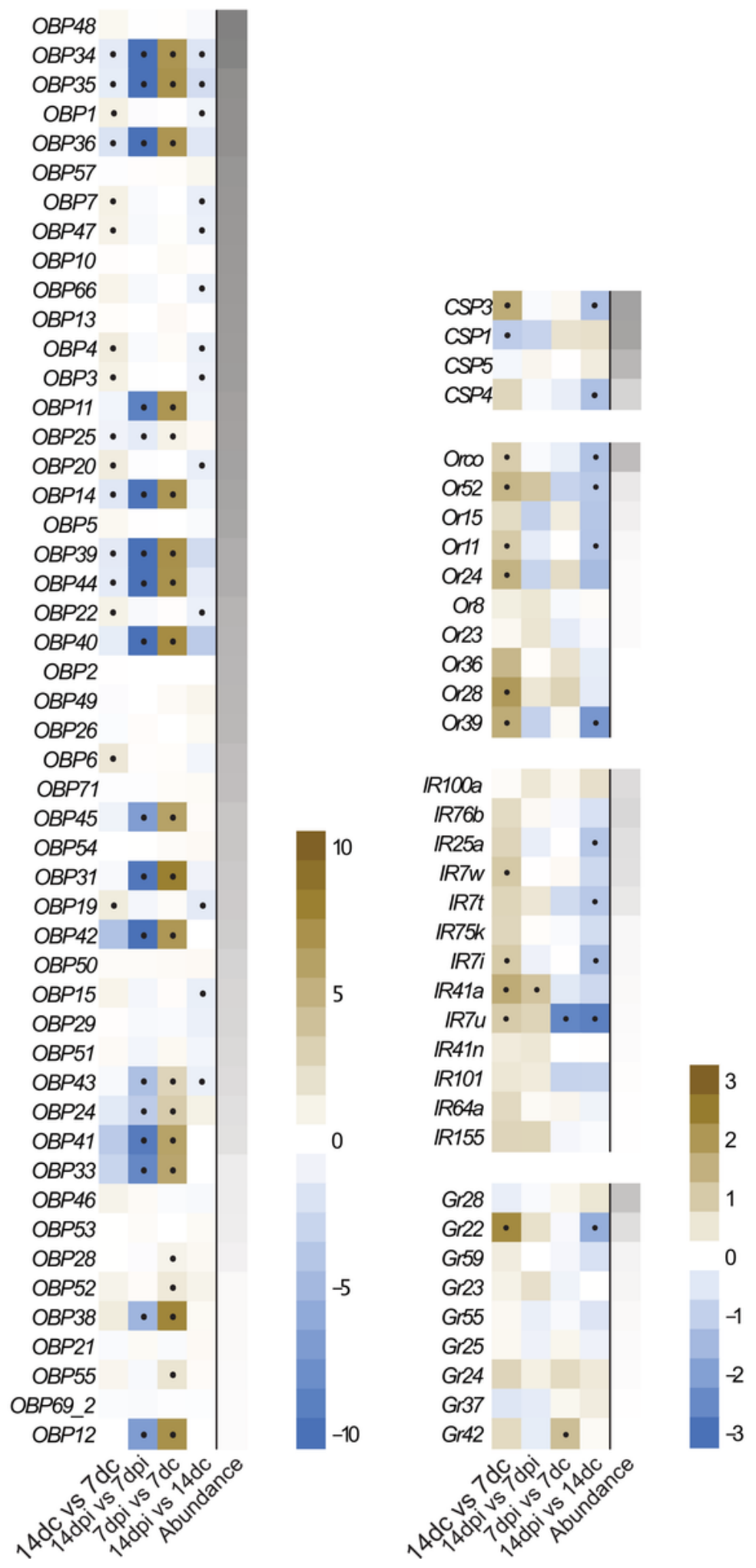




\section{Figure 4}

Antennal chemosensory transcript abundance in female Anopheles gambiae is affected by Plasmodium falciparum infection stage. Chemosensory transcripts demonstrating reliable expression (>1 TPM) in the antennae of infected ( 7 days post-infection; dpi) and infectious ( $14 \mathrm{dpi}$ ) female mosquitoes, along with their age-matched controls ( 7 days control; $7 \mathrm{dc}$ and $14 \mathrm{dc}$ ) were analysed in pairwise comparisons displayed as log2 fold change in abundance. Chemosensory genes are arranged into gene families, odorant binding proteins (OBPs), chemosensory proteins (CSPs), odorant receptors (Ors), ionotropic receptors (Irs) and gustatory receptors (Grs), in descending order of abundance (grey gradient bar, log10 TPM). Significant differences in abundance (fold change $>2$; FDR-corrected $p<0.05$ ) are indicated by black dots.

\section{Supplementary Files}

This is a list of supplementary files associated with this preprint. Click to download.

- Supplementaryinformation101121.pdf 